\title{
Estimativa da precipitação excedente em Ipojuca, PE
}

\author{
Francisco de A. S. de Sousa ${ }^{1}$
}

\begin{abstract}
RESUMO
A infiltração é um dos processos do ciclo hidrológico que tem sido exaustivamente estudado e documentado. Geralmente, as teorias e modelos propostos para a descrição desse processo são aplicados a pequenos lotes de terra, sujeitos a intensidade de chuva maior que sua capacidade de infiltração. O objetivo deste trabalho foi estimar o excesso de chuva para dois eventos de precipitação pluvial, fornecendo-se o valor inicial do conteúdo de umidade da camada superior do solo e os parâmetros da taxa mínima de capacidade de infiltração, $\mathrm{f}_{\mathrm{c}}$ e de capacidade de infiltração inicial, $\mathrm{f}_{0}$. $\mathrm{O}$ excesso de chuva produzida no período total no primeiro caso, foi de $15,2 \mathrm{~mm}$ e de $8,2 \mathrm{~mm}$ no segundo.
\end{abstract}

Palavras-chave: capacidade de infiltração, escoamento superficial, precipitação efetiva

\section{Estimation of precipitation surplus in Ipojuca, PE}

\begin{abstract}
Infiltration is one of the processes of the hydrologic cycle that has been exhaustively studied and documented. The theories and models proposed for the description of these processes are generally applied for small plots of land, subjected to an intensity of rainfall greater than its infiltration capacity. The objective of this study was to estimate the excess of rainfall for two events of the pluvial precipitation, providing the initial value of the water content of the upper soil layer and the parameters like minimum rate of infiltration capacity $\left(\mathrm{f}_{\mathrm{c}}\right)$ and initial infiltration capacity $\left(\mathrm{f}_{\mathrm{o}}\right)$. The excess of rainfall produced during the total period was $15.2 \mathrm{~mm}$ in the first case, and $8.2 \mathrm{~mm}$ in the second.
\end{abstract}

Key words: infiltration capacity, runoff, effective rainfall 


\section{INTRODUÇÃO}

A infiltração não é apenas um processo de entrada de água no solo, mas também parte significativa do ciclo hidrológico, visto que determina a divisão de água fornecida à bacia hidrográfica na forma de chuva, em duas partes, uma que entra na superfície do solo para recarga da umidade das camadas superior e inferior do solo e outra parte é a que permanece na superfície da bacia para produzir o escoamento superficial direto na rede de drenagem. Os principais fatores que intervem no processo de infiltração, são: tipo de solo, grau de umidade, efeito de precipitação e cobertura vegetal.

Tan \& O’Connor (1996) propuseram uma equação empírica que relaciona a taxa de infiltração ao conteúdo real de umidade do solo, para incorporá-la ao modelo Soil Moisture Accounting and Routing (SMAR). Após essa incorporação o modelo foi chamado de SMARY; o Y se refere à taxa de infiltração da equação adicionada ao modelo SMAR, desenvolvido e testado em quatro bacias hidrográficas com diferentes condições climáticas: úmida, semi-árida e seca. Os resultados do modelo SMARY se mostraram superiores aos do SMAR, em termos de coeficiente de determinação, erro médio quadrático e erro relativo. O modelo modificado fornece uma interpretação mais racional do processo físico da infiltração, além de facilitar a determinação da taxa de infiltração real sob condições específicas de umidade do solo. A infiltração exerce importante papel na geração do escoamento superficial e é o primeiro mecanismo que a controla; é, também, uma componente por excelência no processo chuva-vazão.

Técnicas para modelar esta componente tem variado de taxas médias para tipos específicos de solo e cobertura vegetal a soluções teóricas de equações diferenciais parciais para fluxo em meio poroso. Os trabalhos de Morel-Seytoux (1978) e Smith \& Parlange (1978) são alguns exemplos de modelos matemáticos para solos não-saturados. Aplicações práticas desses modelos sofisticados são, muitas vezes, limitadas à disponibilidade de dados de entrada.

A equação de Horton (1940) foi proposta, originalmente, para descrever a infiltração quando o suprimento de água à superfície não é limitado; caso contrário, elas falham ao simular a infiltração sob condições de chuvas variáveis, exceto para tempestade forte, quando a intensidade da chuva sempre excede a taxa de infiltração potencial (ou seja, a superfície do solo está sempre saturada). Muitos pesquisadores, inclusive Mls (1980) têm proposto modificações dessas equações para serem aplicadas quando, para uma parte da tempestade, a intensidade da precipitação é menor que a taxa potencial de infiltração. A consideração básica é a de que, para dado tempo e solo e condições de umidade inicial, a taxa potencial de infiltração é determinada unicamente pela infiltração acumulada até esse tempo. Christiaens \& Feyen (2000) usaram três diferentes metodologias para estimar as propriedades hidráulicas do solo, que servem como entrada dos modelos hidrológicos distribuídos com base física. Hsu et al. (2002) utilizaram as fórmulas de Horton, Philip e Green-Ampt para avaliar a capacidade de infiltração da água em três tipos de solo. Angulo-Jaramillo et al. (2003) apresentam duas soluções para a equação de infiltração de água no solo, em que uma delas despreza os efeitos da força da gravidade. Islam et al. (2006) utilizaram métodos diretos e indiretos de estimativa dos valores dos parâmetros hidráulicos do solo, usados como entrada dos modelos hidrológicos para simular variáveis de balanço hídrico, como conteúdo de água no solo. Mollerup \& Hansen (2007) fizeram comparações de desempenho de um modelo para estimativa de infiltração da água no solo com o modelo de Parlange e o método do infiltrômetro a fim de determinar a condutividade hidráulica saturada. Shi et al. (2009) utilizam o modelo Soil Conservation Service Curve Number (SCS-CN) para predizer o excesso de chuva na bacia experimental Three Gorges, na China. Weill et al. (2009) fizeram uso de uma modelagem integrada superfície-subsuperfície, com base na equação de Richards, para determinar o escoamento superficial após os processos de perdas no âmbito da bacia hidrográfica. Omuto \& Gumbe (2009) discutem a utilização de um software livre denominado HydroMe para estimativa rápida e eficiente de parâmetros hidráulicos do solo, comumente usados nos modelos de infiltração e retenção de água. Wang et al. (2009) apresentaram uma solução algébrica unidimensional para estudar o processo de infiltração e redistribuição em solo não saturado, com base na equação Richards. Moleraup et al. (2008) propuseram uma rotina inversa combinada, para o Modelo Campbell-Mualem, a fim de avaliar os parâmetros hidráulicos do solo.

O estudo da infiltração é extensivamente coberto pela literatura técnica e científica mas sua aplicação é tratada em livros textos e manuais de hidrologia, em capítulos separados. Apesar da abundância de informações a respeito do tema, existem muitos tópicos que não são adequadamente tratados, que são: variação da capacidade de infiltração durante períodos com baixa intensidade de precipitação, tempo de retenção para taxas de precipitação constante ou variável (em inglês, time of ponding) e a estimativa da chuva excedente sobre partes relativamente grandes de uma bacia hidrográfica.

O objetivo deste trabalho é apresentar um modelo conceitual simples e capaz de responder a alguns desses problemas relacionados ao processo de infiltração e estimar o excesso de chuva para eventos de chuvas específicos.

\section{MATERIAL E MÉTODOS}

Toda discussão relativa à infiltração, deveria iniciar com a definição de dois termos já bem conhecidos mas muitas vezes confundidos, isto é, taxa de capacidade de infiltração e taxa de infiltração real; esta última é o volume de água que entra no solo por unidade de área e tempo; já a capacidade de infiltração do solo de determinado local e instante específico é definida como a mais alta taxa possível de água que entra no solo; só é possível conhecer essa taxa se o suprimento de água no local for maior ou igual a ela; se, porém, este suprimento, geralmente em forma de chuva, for menor, então a taxa de infiltração real será igual à taxa de suprimento e não igual à taxa de capacidade de infiltração; esta última taxa só será igual à taxa real de infiltração se a taxa de precipitação for maior ou igual a ela. 
As taxas de capacidade e real de infiltração variam durante a precipitação e por algum tempo, após o evento de chuva. Em geral, essas variações são descritas em função do tempo mas alguns imaginam que nesses processos a mera passagem do tempo não se pode constituir em mudanças nos valores dessas variáveis. A capacidade de infiltração depende do número de fatores relacionados ao tipo de solo e à cobertura vegetal; no entanto, para certo local a capacidade de infiltração depende, em muitos casos, do conteúdo de umidade da camada superior do solo; este conteúdo de umidade não é constante, ou seja, ele muda durante o evento de precipitação dependendo de dois fatores: o primeiro é a infiltração real, função da distribuição de intensidade da precipitação e das mudanças dos valores da capacidade de infiltração enquanto o segundo fator é a taxa de percolação de umidade da camada superior para as camadas inferiores do solo. A taxa de percolação também está sujeita aos tipos de solo do local considerado e do conteúdo de umidade da camada superior do solo; para certo local, essa taxa é função do conteúdo de umidade.

O modelo compreende dois elementos mostrados esquematicamente na Figura 1, em que um é o armazenamento, que recebe uma entrada e produz uma saída; o outro é um elemento regulador, que também recebe uma entrada mas produz duas saídas; cada elemento tem uma variável de estado que determina a magnitude de suas saídas; os dois elementos são ligados por realimentação para transmitir informações acerca do estado do elemento armazenamento para o elemento regulador; esses dois elementos são também relacionados pelo fato de que uma das saídas do elemento regulador é entrada do elemento armazenamento.

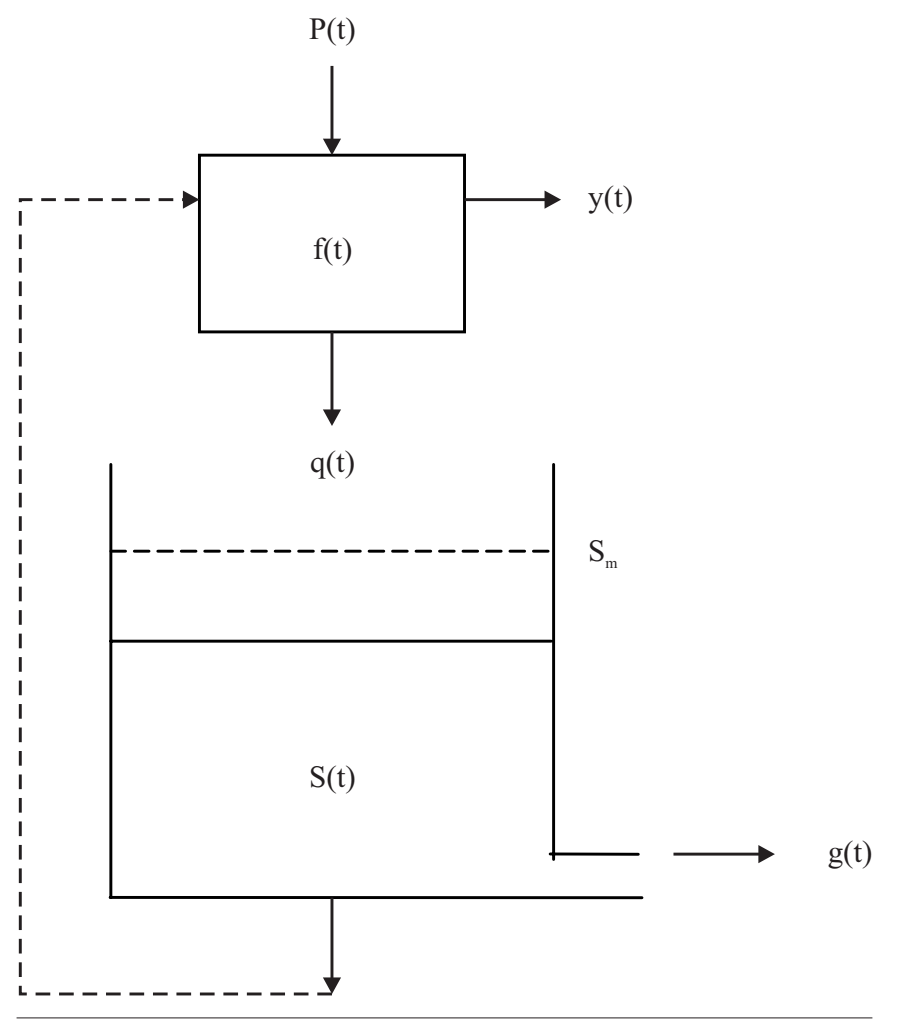

Figura 1. Estrutura esquemática do modelo Diskin-Nazimov
Os valores das duas variáveis de estado e das várias entradas e saídas dependem, obviamente, da forma de entrada no modelo, que é também a entrada para o elemento regulador; referida entrada é dada como função do tempo na forma de hietograma, usando-se um intervalo de tempo fixo; consequentemente, as outras variáveis são também apresentadas em função do tempo e a computação é realizada para o mesmo intervalo de tempo fixo. A computação do modelo se inicia para um instante adotado como tempo zero com um valor da variável de estado do elemento armazenamento conhecido ou arbitrado; tal computação produz valores para várias saídas e para variáveis de estado no final de cada intervalo de tempo, a partir de valores conhecidos do armazenamento e da entrada do modelo, no início desse intervalo de tempo.

\section{Descrição do modelo}

A Figura 1 exibe a estrutura do modelo Diskin-Nazimov, em que $\mathrm{P}(\mathrm{t})$ - intensidade da chuva, $\mathrm{f}(\mathrm{t})$ - capacidade de infiltração, $\mathrm{q}(\mathrm{t})$ - taxa de infiltração na camada superior do solo, S(t) - umidade na camada superior do solo, Sm - valor máximo da umidade do solo, g(t) - taxa de percolação na camada superior do solo e $\mathrm{y}(\mathrm{t})$ - intensidade do excesso de chuva.

Nota-se que o modelo inclui dois elementos principais: um é o elemento armazenamento, que recebe uma entrada e produz uma saída; o outro é o elemento regulador, que recebe uma entrada e produz duas saídas; cada um dos elementos tem uma variável de estado para determinar a magnitude de suas saídas; os elementos são relacionados por um processo de realimentação a fim de transmitir informações acerca do estado do elemento armazenamento para o elemento regulador.

A variável de estado do elemento armazenamento é o volume de água armazenada nesse elemento e é expresso em unidade de volume por unidade de área ou profundidade e seu valor, em qualquer instante t, é denotado por S(t). O elemento armazenamento é assumido como um reservatório linear e produz uma saída proporcional ao volume armazenado. Para qualquer instante t, o valor da saída g(t) é dado de acordo com Diskin \& Nazimov (1996) por:

$$
\mathrm{g}(\mathrm{t})=\mathrm{AS}(\mathrm{t})
$$

em que A é um parâmetro do modelo e g(t) é dado em unidade de volume por unidade de área, por unidade de tempo ou altura por unidade de tempo.

Assume-se que o valor da variável de estado do elemento regulador, em qualquer instante, $\mathrm{f}(\mathrm{t})$, é determinado pelo valor de $\mathrm{S}(\mathrm{t})$ e transmitido para esse elemento através do processo de realimentação. A relação entre as duas variáveis de estado é linear e decrescente:

$$
f(t)=B-C S(t)
$$

em que B e C são parâmetros do modelo.

As saídas do elemento regulador dependem do valor da variável de estado, $\mathrm{f}(\mathrm{t})$, e do valor da entrada, $\mathrm{P}(\mathrm{t})$ no instante 
considerado; as duas saídas q(t) e y(t) são definidas pela seguinte relação:

$$
\begin{aligned}
& \text { Se } \quad \mathrm{P}(\mathrm{t})<\mathrm{f}(\mathrm{t}) \quad \text { então } \quad \mathrm{q}(\mathrm{t})=\mathrm{P}(\mathrm{t}) \quad \text { e } \quad \mathrm{y}(\mathrm{t})=0 \\
& \text { Se } \quad P(t)>f(t) \quad \text { então } \quad q(t)=f(t) \quad \text { e } \quad y(t)=P(t)-f(t)
\end{aligned}
$$

As duas saídas $\mathrm{q}(\mathrm{t})$ e $\mathrm{y}(\mathrm{t})$, a entrada $\mathrm{P}(\mathrm{t})$ e a variável de estado $\mathrm{f}(\mathrm{t})$ são expressas em unidades de volume por unidade de área e tempo ou altura por unidade de tempo.

O valor da variável de estado do elemento armazenamento $\mathrm{S}(\mathrm{t})$ em qualquer instante $\mathrm{t}$, muda a uma taxa que depende da magnitude da entrada e da saída do elemento armazenamento; a relação entre as três variáveis é a expressão do princípio de conservação de massa:

$$
q(t)-g(t)=\frac{d S}{d t}
$$

Ressalta-se que, apesar do uso de relação linear para descrição dos elementos, o modelo é não-linear e, nesse particular, ele não possui a propriedade de sobreposição de respostas para entradas individuais na formação de saída, devido à soma das funções de entrada.

O conteúdo de umidade na zona superior do solo, representado pela altura de água $\mathrm{S}(\mathrm{t})$ no elemento armazenamento, é limitado à faixa:

$$
0 \leq \mathrm{S}(\mathrm{t}) \leq \mathrm{S}_{\mathrm{m}}
$$

em que $S_{m}$ é um parâmetro do modelo e determina a altura máxima de $\mathrm{S}(\mathrm{t})$. A relação entre entrada, saída e volume de água no elemento armazenamento, é descrita pelas Eqs. 1 a 5; está claro que o acréscimo no conteúdo de umidade do elemento armazenamento na faixa definida pela Eq. 6, causa decréscimo na capacidade de infiltração e acréscimo na taxa de percolação; já o conteúdo de umidade do elemento armazenamento cresce enquanto q(t) > g(t), Eq. 5, e seu valor máximo é alcançado quando a entrada para este elemento é igual à saída, ou seja:

$$
\mathrm{S}(\mathrm{t})=\mathrm{S}_{\mathrm{m}} \text { quando } \mathrm{q}(\mathrm{t})=\mathrm{g}(\mathrm{t})=\mathrm{f}_{\mathrm{c}} \text { e } \frac{\mathrm{dS}}{\mathrm{dt}}=0
$$

em que $\mathrm{f}_{\mathrm{c}}$ é um parâmetro do modelo e representa a menor taxa de infiltração ou a maior taxa de percolação. Essas taxas são alcançadas após um evento de chuva prolongado com intensidade maior ou igual a menor taxa de capacidade de infiltração.

O elemento regulador representa a superfície ou a zona superior do solo, que determina a capacidade de infiltração média, f(t), da área considerada; a entrada para este elemento é feita de acordo com o conteúdo de umidade na zona superior do solo; quando a umidade for completamente deplecionada, $\mathrm{S}(\mathrm{t})=0$, a capacidade de infiltração se encontrará no seu valor máximo, $\mathrm{f}(\mathrm{t})=\mathrm{f}_{0}$; em caso contrário, quando a umidade alcançar o valor máximo, $\mathrm{S}(\mathrm{t})=\mathrm{S}_{\mathrm{m}}$, a capacidade de infiltração se encontrará no seu valor mínimo, $\mathrm{f}(\mathrm{t})=\mathrm{f}_{\mathrm{C}}$; enfim, a taxa de capacidade de infiltração máxima, $\mathrm{f}_{0}$, tam- bém é um parâmetro do modelo.

O elemento regulador determina também a divisão da entrada da chuva, $\mathrm{P}(\mathrm{t})$, em infiltração real, $\mathrm{q}(\mathrm{t})$ e em excesso de chuva, $\mathrm{y}(\mathrm{t})$; este processo é realizado de acordo com o valor presente da capacidade de infiltração, $f(t)$, especificado pelas Eqs. 3 e 4.

\section{Definição dos parâmetros}

Conforme comprovado no item anterior, a descrição da aplicação do modelo envolve três parâmetros: armazenamento máximo, $\mathrm{S}_{\mathrm{m}}$, capacidades inicial, $\mathrm{f}_{0}$ e final $\mathrm{f}_{\mathrm{c}}$, de infiltração; trata-se de parâmetros relacionados a A, B e C, usados para definir os dois elementos do modelo nas Eqs. 1 e 2; a relação entre as duas séries de parâmetros é dada como segue; a taxa máxima de percolação, $\mathrm{g}_{\mathrm{c}}=\mathrm{f}_{\mathrm{c}}$, é obtida quando o elemento armazenamento se encontra no seu valor máximo, $\mathrm{S}_{\mathrm{m}}$; substituindo-se esses valores na Eq. 1 tem-se:

$$
A=\frac{f_{c}}{S_{m}}
$$

comparando-se o valor máximo da capacidade de infiltração, $\mathrm{f}_{0}$, obtido quando $\mathrm{S}(\mathrm{t})=0$, com o valor dado pela Eq. 2 tem-se:

$$
B=f_{o}
$$

Agora, considerando-se a igualdade de $\mathrm{f}(\mathrm{t})$ e $\mathrm{g}(\mathrm{t})$ quando $\mathrm{S}(\mathrm{t})=\mathrm{S}_{\mathrm{m}}$ e se usando as Eqs. 1 e 2 tem-se:

$$
\mathrm{AS}_{\mathrm{m}}=\mathrm{B}-\mathrm{CS}_{\mathrm{m}}
$$

substituindo-se as Eqs. 8 e 9 na Eq. 10 e se resolvendo para C, tem-se:

$$
C=\frac{\left(f_{o}-f_{c}\right)}{S_{m}}
$$

Usando-se esses resultados, as Eqs. 1 e 2 podem ser reescritas como:

$$
\begin{aligned}
& g(t)=f_{c} \frac{S(t)}{S_{m}} \\
& f(t)=f o-\left(f_{o}-f_{c}\right) S(t) / S_{m}
\end{aligned}
$$

\section{Método computacional}

Pode-se aplicar este modelo para calcular as variações da capacidade de infiltração e a produção do excesso de chuva durante e após um evento de chuva, descrito pelo hietograma. Assume-se que os valores dos parâmetros do modelo para a área considerada são conhecidos e o valor inicial da variável de estado do elemento armazenamento é especificado. Usando-se as Eqs. 12 e 13 determinam-se os valores iniciais das taxas de percolação e de capacidade de infiltração e os cálculos são realizados para sucessivos intervalos de tempo, iguais ou não.

Considerando-se o intervalo de tempo $\left(\Delta t=t_{i+1}-t_{i}\right)$, a 
Eq. 5 pode ser reescrita da seguinte forma:

$$
\mathrm{S}_{\mathrm{f}}-\mathrm{S}_{\mathrm{i}}=\left(\mathrm{q}_{\mathrm{i}}-\mathrm{q}_{\mathrm{f}}\right) \Delta \mathrm{t} / 2-\left(\mathrm{g}_{\mathrm{i}}-\mathrm{g}_{\mathrm{f}}\right) \Delta \mathrm{t} / 2
$$

Os índices nas variáveis da Eq. 14 se referem ao início e ao final do intervalo.

Com base nas definições da infiltração real, q, nas Eqs. 3 e 4 e se denotando a intensidade de chuva durante o intervalo por $\mathrm{P}$, três casos são possíveis:

Caso (a): a intensidade de chuva é maior que a capacidade de infiltração no início e no final do intervalo de tempo: $P>f_{i}$ e $P>f_{f}$, caso em que: $q_{i}=f_{i}$ e $q_{f}=f_{f}$, substituindo-se na Eq. 14 tem-se:

$$
\mathrm{S}_{\mathrm{f}}-\mathrm{S}_{\mathrm{i}}=\left(\mathrm{f}_{\mathrm{i}}-\mathrm{f}_{\mathrm{f}}\right) \Delta \mathrm{t} / 2-\left(\mathrm{g}_{\mathrm{i}}-\mathrm{g}_{\mathrm{f}}\right) \Delta \mathrm{t} / 2
$$

usando-se as definições de g e f das Eqs. 12 e 13, a última expressão se torna:

$$
\mathrm{S}_{\mathrm{f}}-\mathrm{S}_{\mathrm{f}}=\left[\mathrm{f}_{0}-\left(\mathrm{f}_{0}-\mathrm{f}_{\mathrm{c}}\right)\left(\mathrm{S}_{\mathrm{i}}+\mathrm{S}_{\mathrm{f}}\right) / 2 \mathrm{~S}_{\mathrm{m}}\right] \Delta \mathrm{t}-\mathrm{f}_{\mathrm{c}}\left(\mathrm{S}_{\mathrm{i}}+\mathrm{S}_{\mathrm{f}}\right) \Delta \mathrm{t} / 2 \mathrm{~S}_{\mathrm{m}}
$$

Rearranjando-se os termos e se resolvendo para $\mathrm{S}_{\mathrm{f}}$, tem-se:

$$
\mathrm{S}_{\mathrm{f}}=\left[\mathrm{f}_{\mathrm{o}} \Delta \mathrm{t}+\mathrm{S}_{\mathrm{i}}\left(1-\mathrm{f}_{\mathrm{o}} \Delta \mathrm{t} / 2 \mathrm{~S}_{\mathrm{m}}\right)\right] /\left(1+\mathrm{f}_{\mathrm{o}} \Delta \mathrm{t} / 2 \mathrm{~S}_{\mathrm{m}}\right)
$$

Caso (b): a intensidade de chuva é menor que a capacidade de infiltração no início e no final do intervalo de tempo: $\mathrm{P}<\mathrm{f}_{\mathrm{i}}$ e $\mathrm{P}<\mathrm{f}_{\mathrm{f}}$, caso em que: $\mathrm{P}=\left(\mathrm{q}_{\mathrm{i}}+\mathrm{q}_{\mathrm{f}}\right) / 2$ e a $\mathrm{Eq}$. 14 pode ser reescrita como:

$$
\mathrm{S}_{\mathrm{f}}-\mathrm{S}_{\mathrm{i}}=\mathrm{P} \Delta \mathrm{t}-\left(\mathrm{g}_{\mathrm{i}}+\mathrm{g}_{\mathrm{f}}\right) \Delta \mathrm{t} / 2
$$

através da definição de g na Eq. 12 a última expressão se torna:

$$
\mathrm{S}_{\mathrm{f}}-\mathrm{S}_{\mathrm{i}}=\mathrm{P} \Delta \mathrm{t}-\mathrm{f}_{\mathrm{c}}\left(\mathrm{S}_{\mathrm{i}}+\mathrm{S}_{\mathrm{f}}\right) \Delta \mathrm{t} / 2 \mathrm{~S}_{\mathrm{m}}
$$

Rearranjando-se os termos e se resolvendo para $\mathrm{S}_{\mathrm{f}}$ conduz a:

$$
\mathrm{S}_{\mathrm{f}}=\left[\mathrm{S}_{\mathrm{i}}\left(1-\mathrm{f}_{\mathrm{c}} \Delta \mathrm{t} / 2 \mathrm{~S}_{\mathrm{m}}\right)+\mathrm{R} \Delta \mathrm{t}\right] /\left(1+\mathrm{f}_{\mathrm{c}} \Delta \mathrm{t} / 2 \mathrm{~S}_{\mathrm{m}}\right)
$$

Caso (c): a intensidade de chuva é menor que a capacidade de infiltração no início do intervalo de tempo e maior no final: $\mathrm{P}<\mathrm{f}_{\mathrm{i}}$ e $\mathrm{P}<\mathrm{f}_{\mathrm{f}}$; neste caso, a capacidade de infiltração será igual à taxa de precipitação, durante algum tempo, dentro do intervalo de tempo; o conteúdo de umidade do elemento armazenamento nesse instante, denotado por $S_{r}$, é obtido fazendo $f(t)=P$ na Eq. 13 e se resolvendo para $S(t)$ :

$$
\mathrm{S}_{\mathrm{r}}=\left(\mathrm{f}_{0}-\mathrm{P}\right) \mathrm{S}_{\mathrm{m}} /\left(\mathrm{f}_{0}-\mathrm{f}_{\mathrm{c}}\right)
$$

Obter-se-ão o tempo em que essa igualdade ocorrerá, relativo ao início do intervalo de tempo de computação, fazendo-se $\mathrm{S}_{\mathrm{f}}=\mathrm{S}_{\mathrm{r}}$ e $\Delta \mathrm{t}=\Delta \mathrm{t}_{1}$, substituindo-a na Eq. 19 e se resolvendo para $\Delta \mathrm{t}_{1}$ :

$$
\Delta \mathrm{t}_{1}=\left(\mathrm{S}_{\mathrm{r}}-\mathrm{S}_{\mathrm{i}}\right) /\left[\mathrm{R}-\left(\mathrm{S}_{\mathrm{i}}+\mathrm{S}_{\mathrm{r}}\right) \mathrm{f}_{\mathrm{c}} / 2 \mathrm{~S}_{\mathrm{m}}\right]
$$

obviamente, $0<\Delta \mathrm{t}_{1}<\Delta \mathrm{t}$. O conteúdo do elemento armazenamento no final do intervalo de tempo de computação é obtido com a Eq. 17 com $\Delta \mathrm{t}_{2}=\Delta_{\mathrm{t}}-\Delta \mathrm{t}_{1}$ substituindo-se $\Delta_{\mathrm{t}}, \mathrm{S}_{\mathrm{r}}$ e $\mathrm{S}_{\mathrm{i}}$; obtém-se, assim, a expressão:

$$
\mathrm{S}_{\mathrm{f}}=\left[\mathrm{f}_{\mathrm{O}} \Delta \mathrm{t}_{2}+\mathrm{S}_{\mathrm{r}}\left(1-\mathrm{f}_{\mathrm{o}} \Delta \mathrm{t}_{2} / 2 \mathrm{~S}_{\mathrm{m}}\right)\right] /\left(1+\mathrm{f}_{\mathrm{O}} \Delta \mathrm{t}_{2} / 2 \mathrm{~S}_{\mathrm{m}}\right)
$$

Vê-se que, para determinado local representado pelos parâmetros do modelo e para um intervalo de tempo constante, as Eqs. 17 e 20, obtidas para os casos (a) e (b), respectivamente, podem ser simplificadas pelas seguintes equações recursivas:

$$
\mathrm{S}_{\mathrm{f}}=\mathrm{K}_{1} \mathrm{~S}_{\mathrm{i}}+\mathrm{K}_{2}
$$

para o Caso (a) e,

$$
\mathrm{S}_{\mathrm{f}}=\mathrm{K}_{3} \mathrm{~S}_{\mathrm{i}}+\mathrm{K}_{4} \mathrm{P}
$$

para o Caso (b). Os coeficientes $K_{1}, K_{2}, K_{3}$ e $K_{4}$ são constantes computadas a partir dos valores dos parâmetros do modelo e do valor do intervalo de tempo. Comparando-se as Eqs. 24 e 25 com as Eqs. 17 e 20, respectivamente, obterse-ão as seguintes expressões para os coeficientes:

$$
\begin{aligned}
& \mathrm{K}_{1}=\left(1-\mathrm{f}_{\mathrm{o}} \Delta \mathrm{t} / 2 \mathrm{~S}_{\mathrm{m}}\right) /\left(1+\mathrm{f}_{\mathrm{o}} \Delta \mathrm{t} / 2 \mathrm{~S}_{\mathrm{m}}\right) \\
& \mathrm{K}_{2}=\mathrm{f}_{\mathrm{o}} \Delta \mathrm{t} /\left(1+\mathrm{f}_{\mathrm{o}} \Delta \mathrm{t} / 2 \mathrm{~S}_{\mathrm{m}}\right) \\
& \mathrm{K}_{3}=\left(1-\mathrm{f}_{\mathrm{c}} \Delta \mathrm{t} / 2 \mathrm{~S}_{\mathrm{m}}\right) /\left(1+\mathrm{f}_{\mathrm{c}} \Delta \mathrm{t} / 2 \mathrm{~S}_{\mathrm{m}}\right) \\
& \mathrm{K}_{4}=\Delta \mathrm{t}\left(1+\mathrm{f}_{\mathrm{c}} \Delta \mathrm{t} / 2 \mathrm{~S}_{\mathrm{m}}\right)
\end{aligned}
$$

\section{Tempo de retenção da precipitação e excesso de chuva}

Tempo de retenção da chuva ou 'ponding', $t_{p}$, é definido, em estudos de infiltração, como o início da formação de excesso de chuva sobre a superfície da área considerada; o $t_{p}$ sinaliza o tempo no qual a taxa de infiltração real, antes inferior, se iguala à taxa de capacidade de infiltração; geralmente, segundo Smith \& Parlange (1978), o termo $t_{p}$ se refere a eventos no qual a camada superior do solo apresenta armazenamento inicial igual a zero e intensidade de chuva, P, constante, sempre menor que a capacidade de infiltração máxima e maior que a capacidade mínima:

$$
\mathrm{f}_{\mathrm{c}}<\mathrm{P}<\mathrm{f}_{\mathrm{O}}
$$

Para evento de chuvas com intensidades constantes na faixa especificada pela Eq. 30 existe apenas um $t_{p}$, e pode ser computado pela Eq. 5 de conservação de massa; se, porém, a intensidade da chuva não for constante, é provável que haja mais do que um $t_{p}$, dependendo da forma do hieotograma; esses $t_{p}$ pertencem a um dos dois tipos possíveis, descritos a seguir: o primeiro tipo apresenta $t_{p}$ com valores inteiros e múltiplos do intervalo de tempo usado no hietograma e ocorrem quando a intensidade de chuva, antes abaixo, supera a capacidade de infiltração; já no segundo tipo 
os valores de $t_{p}$ ocorrem porque a taxa de capacidade de infiltração varia no intervalo de tempo de um valor maior que a intensidade de chuva para um menor. A ocorrência de um $t_{p}$ do primeiro tipo significa que o excesso de precipitação será produzido por, pelo menos, um intervalo de tempo seguinte a $t_{p}$; para $t_{p}$ do segundo tipo, o excesso de precipitação será produzido pelo menos em um pequeno intervalo de $\mathrm{Dt}_{2}$ entre $\mathrm{t}_{\mathrm{p}}$ e o fim do intervalo de tempo da computação; em ambos os casos, a possibilidade de que a produção de excesso de chuva continuará por um ou mais intervalos de tempo adicional, depende da forma do hietograma da chuva nesses intervalos de tempo.

A altura do excesso de chuva, $\Delta \mathrm{P}_{\mathrm{x}}$, produzido em qualquer intervalo de tempo durante o qual a intensidade de chuva é maior que a capacidade de infiltração, é computada por:

$$
\Delta \mathrm{P}_{\mathrm{x}}=\left[\mathrm{P}-\left(\mathrm{f}_{\mathrm{i}}+\mathrm{f}_{\mathrm{f}}\right) / 2\right] \Delta \mathrm{t}
$$

ou

$$
\Delta \mathrm{P}_{\mathrm{x}}=\left[\mathrm{P}-\left(\mathrm{P}+\mathrm{f}_{\mathrm{f}}\right) / 2\right] \Delta \mathrm{t}_{2}
$$

Para intervalos de tempo em que ocorre $t_{p}$ do segundo tipo.

Este modelo não pode ser aplicado em locais onde a redução na taxa de capacidade de infiltração se deve à formação de crosta na superfície do solo ou em locais com presença de solos com camadas inferiores relativamente impermeáveis.

\section{Área de estudo e dados}

Na aplicação do modelo se utilizaram os dados do município de Ipojuca, PE, e os dados de intensidade de chuva foram obtidos nas plataformas de coleta de dados - PCDs, do Laboratório de Meteorologia de Pernambuco/Instituto Tecnológico do Estado de Pernambuco - LAMEPE/ITEP. As principais características físicas da área estudada são: área de $507 \mathrm{~km}^{2}$, solo areno-argiloso, relevo ondulado e cobertura vegetal de floresta subperenifólia.

\section{RESULTADOS E DISCUSSÃO}

O modelo descrito para estimar o processo de infiltração e excesso de precipitação é simples e faz uso de duas variáveis de estado: capacidade de infiltração, $f(t)$ e variação de armazenamento de água no solo, $\mathrm{S}(\mathrm{t})$ e três parâmetros relacionados às propriedades físicas da camada superior do solo: capacidade máxima e mínima de infiltração, $\mathrm{f}_{o}$ e $\mathrm{f}_{\mathrm{c}}$ e armazenamento máximo de umidade do solo. No processo de estimativas é necessário, ainda, adotar um intervalo de tempo e expressar a chuva na forma de hietograma.

Os cálculos são realizados pelas Eqs. recursivas 24 e 25. Faz-se uso, na metodologia aqui aplicada, do valor da variável de estado armazenamento no início do intervalo de tempo e do valor da intensidade da chuva durante este intervalo, para computar o valor dessa variável no final do intervalo; os resultados expostos nas Tabelas 1 e 2 e Figuras 2 e 3, demonstram os efeitos de diferentes estados iniciais no modelo.
Na aplicação se adotaram os seguintes valores para os parâmetros do modelo: $\mathrm{f}_{\mathrm{o}}=70,0 \mathrm{~mm} \mathrm{~h}^{-1}, \mathrm{f}_{\mathrm{c}}=12,0 \mathrm{~mm} \mathrm{~h}^{-1} \mathrm{e}$ $\mathrm{S}_{\mathrm{m}}=65 \mathrm{~mm}$. Duas séries de cálculo são produzidas para dois valores assumidos de conteúdo inicial de umidade na zona superior do solo; o hietograma da chuva e os valores dos parâmetros do modelo foram mantidos constantes nas duas séries de cálculo.

Os valores das intensidades da chuva durante o evento estão listados na segunda coluna da Tabela 1 e o total precipitado foi de $141,1 \mathrm{~mm}$.

Usando-se os valores dos parâmetros supracitados nas Eqs. 12 e 13 obtem-se as expressões para a percolação e infiltração, em função de $\mathrm{S}(\mathrm{t})$, que terão valores numéricos atualizados em cada intervalo de tempo, pelas expressões 24 e 25; adotando-se o intervalo de tempo $\Delta \mathrm{t}=0,5 \mathrm{~h}$ e os valores iniciais dos parâmetros se estimaram os valores dos coeficientes $K_{1}=0,5758, K_{2}=27,5758, K_{3}=0,9118$ e $K_{4}=0,4779$ usando-se as Eqs. 26 a 29; para a primeira série de cálculos o valor do conteúdo de umidade inicial na zona superior do solo foi $\mathrm{S}=0,0 \mathrm{~mm}$. A Tabela 1 lista os resultados da aplicação do modelo e nela pode ser visto que o tempo de retenção da chuva ou 'ponding' ocorre em t $=4,5$ h, que é um valor múltiplo do intervalo de tempo utilizado na computação; na produção do excesso de chuva (Pexc), sétima coluna da Tabela 1, verifica-se, em três intervalos de tempo, $t=4,5$ a 5,5 h e t $=6,5$ h. Nota-se que tal produção se verifica quando a intensidade da chuva é maior que a capacidade de infiltração; fora do intervalo e tempo acima relatados, a intensidade da chuva é menor que a capacidade de infiltração, caso em que os cálculos são realizados usando-se a Eq. 25 para o Caso b, descrito no subcapítulo método computacional. A altura do excesso de chuva produzida em 1,5 h foi 13,3 mm e em todo o período foi igual a 15,2 $\mathrm{mm}$.

Tabela 1. Cálculos da capacidade de infiltração (f) e excesso de chuva (Pexc) para $\left(\mathrm{S}_{0}=0 \mathrm{~mm}\right)$

\begin{tabular}{rrrcccc}
\hline $\begin{array}{c}\mathbf{T} \\
(\mathbf{h})\end{array}$ & $\begin{array}{c}\mathbf{P} \\
\left(\mathbf{m m ~ h}^{-1}\right)\end{array}$ & $\begin{array}{c}\mathbf{S} \\
(\mathbf{m m})\end{array}$ & $\begin{array}{c}\mathbf{F} \\
\left(\mathbf{m m ~ h} \mathbf{~}^{-1}\right)\end{array}$ & $\begin{array}{c}\mathbf{G} \\
(\mathbf{m m} \mathbf{~ h}-\mathbf{1})\end{array}$ & $\begin{array}{c}\mathbf{T p} \\
(\mathbf{h})\end{array}$ & $\begin{array}{c}\text { Pexc } \\
(\mathbf{m m})\end{array}$ \\
0,0 & - & 0,0 & 70,0 & 0,0 & & 0,0 \\
0,5 & 25,0 & 11,9 & 59,3 & 2,2 & & 0,0 \\
1,0 & 20,3 & 20,6 & 51,6 & 3,8 & & 0,0 \\
1,5 & 15,6 & 26,2 & 46,6 & 4,8 & & 0,0 \\
2,0 & 10,9 & 29,1 & 44,0 & 5,4 & & 0,0 \\
2,5 & 20,8 & 36,5 & 37,4 & 6,7 & & 0,0 \\
3,0 & 18,3 & 42,0 & 32,5 & 7,8 & & 0,0 \\
3,5 & 13,9 & 45,0 & 29,9 & 8,3 & & 0,0 \\
4,0 & 15,0 & 48,2 & 27,0 & 8,9 & & 0,0 \\
4,5 & 32,0 & 59,2 & 17,2 & 10,9 & 4,5 & 5,0 \\
5,0 & 29,1 & 61,7 & 15,0 & 11,4 & & 6,5 \\
5,5 & 18,0 & 63,1 & 13,7 & 11,6 & & 1,8 \\
6,0 & 10,9 & 62,7 & 14,0 & 11,6 & & 0,0 \\
6,5 & 17,5 & 63,7 & 13,2 & 11,8 & & 1,9 \\
7,0 & 9,3 & 62,5 & 14,2 & 11,5 & & 0,0 \\
7,5 & 10,5 & 62,0 & 14,7 & 11,4 & & 0,0 \\
8,0 & 9,3 & 61,0 & 15,6 & 11,3 & & 0,0 \\
8,5 & 5,7 & 58,3 & 17,9 & 10,8 & & 0,0 \\
9,0 & 0,0 & 53,2 & 22,5 & 9,8 & & 0,0 \\
soma & 141,1 & & & & 15,2 \\
\hline
\end{tabular}


A Figura 2 exibe as variações da capacidade de infiltração (f) e percolação (g) sobrepostas ao hietograma da chuva. Os valores de capacidade de infiltração mostram a variação esperada, seus valores decrescem quando a intensidade da chuva é maior que a capacidade de infiltração mínima, fc, e aumentam quando a intensidade da chuva é menor que essa capacidade ou quando pára de chover.

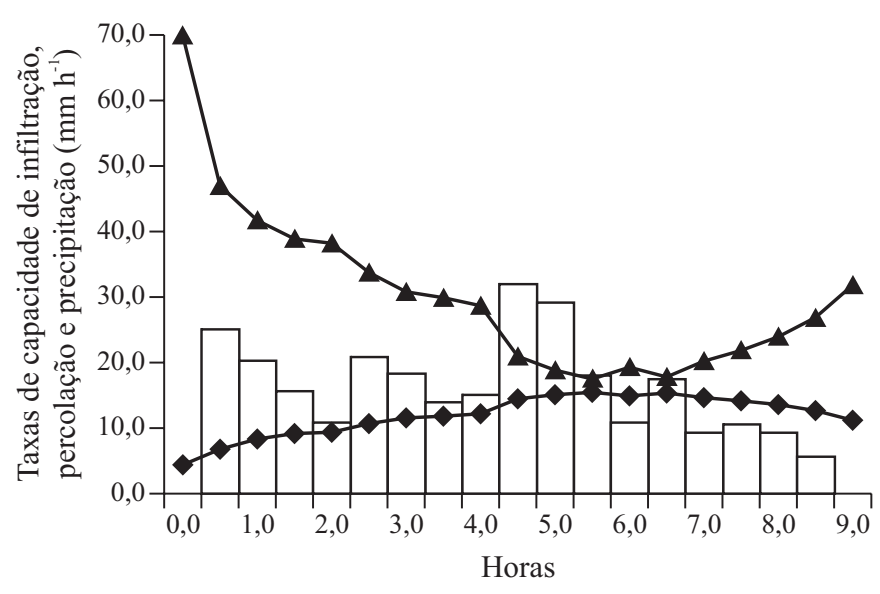

Figura 2. Hietograma $\left(\square\right.$ em $\left.\mathrm{mm} \mathrm{h}^{-1}\right)$, variação da capacidade de infiltração $\left(\bullet\right.$ em $\left.\mathrm{mm} \mathrm{h}^{-1}\right)$ e da percolação $\left(\boldsymbol{\Delta} \mathrm{em} \mathrm{mm} \mathrm{h}^{-1}\right)$ para $\mathrm{S}_{0}=0 \mathrm{~mm}$ como valor inicial

Os resultados da segunda série de cálculos são listados na Tabela 2; os cálculos se iniciaram com os mesmos parâmetros da primeira série de dados; aqui, o valor do conteúdo de umidade inicial na zona superior do solo foi $\mathrm{S}=18,0 \mathrm{~mm}$ e pode ser visto, na Tabela 2, que o tempo de retenção da chuva ou 'ponding' ocorre em $\mathrm{t}=4,5$ h que é, também, um

Tabela 2. Cálculos da capacidade de infiltração (f) e excesso de chuva (Pexc) para $\left(\mathrm{S}_{0}=18 \mathrm{~mm}\right)$

\begin{tabular}{ccccccc}
\hline $\begin{array}{c}\mathbf{T} \\
(\mathbf{h})\end{array}$ & $\begin{array}{c}\mathbf{P} \\
\left(\mathbf{m m ~ h}^{-1}\right)\end{array}$ & $\begin{array}{c}\mathbf{S} \\
(\mathbf{m m})\end{array}$ & $\begin{array}{c}\mathbf{F} \\
\left(\mathbf{m m ~ h}^{-1}\right)\end{array}$ & $\begin{array}{c}\mathbf{G} \\
\left(\mathbf{m m ~ h}^{-1}\right)\end{array}$ & $\begin{array}{c}\mathbf{T p} \\
(\mathbf{h})\end{array}$ & $\begin{array}{c}\text { Pexc } \\
(\mathbf{m m})\end{array}$ \\
0,0 & - & 18,0 & 70,0 & 4,4 & & 0,0 \\
0,5 & 25,0 & 27,7 & 47,0 & 6,8 & & 0,0 \\
1,0 & 20,3 & 34,0 & 41,7 & 8,4 & & 0,0 \\
1,5 & 15,6 & 37,4 & 38,9 & 9,2 & & 0,0 \\
2,0 & 10,9 & 38,2 & 38,2 & 9,4 & & 0,0 \\
2,5 & 20,8 & 43,6 & 33,8 & 10,7 & & 0,0 \\
3,0 & 18,3 & 47,2 & 30,8 & 11,6 & & 0,0 \\
3,5 & 13,9 & 48,2 & 29,9 & 11,9 & & 0,0 \\
4,0 & 15,0 & 49,7 & 28,7 & 12,2 & & 0,0 \\
4,5 & 32,0 & 59,0 & 21,0 & 14,5 & 4,5 & 3,6 \\
5,0 & 29,1 & 61,6 & 18,9 & 15,2 & & 4,6 \\
5,5 & 18,0 & 63,0 & 17,6 & 15,5 & & 0,0 \\
6,0 & 10,9 & 60,8 & 19,5 & 15,0 & & 0,0 \\
6,5 & 17,5 & 62,6 & 18,0 & 15,4 & & 0,0 \\
7,0 & 9,3 & 59,7 & 20,4 & 14,7 & & 0,0 \\
7,5 & 10,5 & 57,8 & 22,0 & 14,2 & & 0,0 \\
8,0 & 9,3 & 55,4 & 23,9 & 13,6 & & 0,0 \\
8,5 & 5,7 & 51,7 & 27,1 & 12,7 & & 0,0 \\
9,0 & 0,0 & 45,7 & 32,0 & 11,2 & & 0,0 \\
soma & 141,1 & & & & 8,2 \\
\hline
\end{tabular}

valor múltiplo do intervalo de tempo utilizado na computação; a produção do excesso de chuva (Pexc), sétima coluna da Tabela 2, ocorre em dois intervalos de tempo, de $t=4,5$ a 5,0 h; essa produção se dá de acordo com as mesmas características da primeira série de cálculos relativas à intensidade da chuva e capacidade de infiltração; enfim, a altura do excesso de chuva produzida em $1,0 \mathrm{~h}$, em todo o período, foi $8,2 \mathrm{~mm}$.

A Figura 3 apresenta as variações da capacidade de infiltração (f) e percolação (g) sobrepostas ao hietograma da chuva. Pode-se notar novamente, que o modelo mostra, de forma satisfatória, a variação esperada para os valores de capacidade de infiltração.

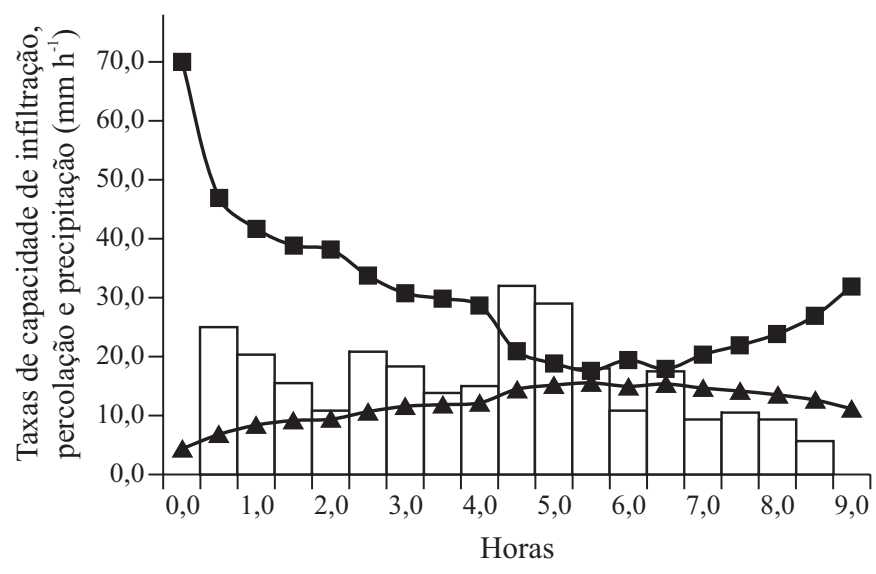

Figura 3. Hietograma ( em $\mathrm{mm} \mathrm{h}^{-1}$ ), variação da capacidade de infiltração $\left(\bullet\right.$ em $\left.\mathrm{mm} \mathrm{h}^{-1}\right)$ e da percolação $\left(\boldsymbol{\Delta}\right.$ em $\mathrm{mm} \mathrm{h}^{-1}$ ) para $\mathrm{S}_{0}=18,0 \mathrm{~mm}$ como valor inicial

\section{CONCLUSÕES}

1. O modelo produz resultados consistentes com o esperado, a priori, e seu uso elimina a necessidade de obtenção da curva de capacidade de infiltração da área em estudo.

2. O modelo produz declínio dos valores de capacidade de infiltração quando a intensidade da chuva é maior que a taxa de capacidade mínima e apresenta acréscimo na capacidade de infiltração quando a intensidade da chuva é muito baixa ou igual a zero.

3. Uma característica importante do modelo é a de que bons resultados são obtidos quando se assume que a infiltração e a percolação dependem do conteúdo de umidade da camada superior do solo.

4. Ao invés de representar a capacidade de infiltração como função do tempo, a mudança de ênfase nesta relação é considerada modelagem importante para compreensão do processo de infiltração.

\section{AGRADECIMENTOS}

Ao CNPq, pela bolsa de Produtividade em Pesquisa concedida ao autor, e ao LAMEPE/ITEP do Estado de Pernambuco, pela cessão dos dados de intensidade de chuva. 


\section{LITERATURA CITADA}

Angulo-Jaramillo, R.; Elrick, D.; Parlange, J. Y.; Gérard-Marchant, P.; Haverkamp, R. Analysis of short-time single-ring infiltration under falling-head conditions with gravitational effects. Hydrology Days, 2003, Fort Collins. Proceedings... Fort Collins: Colorado State University, 2003. p.16-23.

Christiaens, K.; Feyen, J. The influence of different methods to derive soil hydraulic properties on the uncertainty of various model outputs of a distributed hydrological model. Physics and Chemistry of the Earth, Part B: Hydrology, Oceans and Atmosphere, v.25, n.7-8, p.679-683, 2000.

Diskin, M. H; Nazimov, N. Ponding time and infiltration capacity variation during steady rainfall. Journal of Hydrology, v.178, n.2, p.369-380, 1996.

Horton, R. E. An approach toward a physical interpretation of infiltration capacity soil. Science Society of American Proceeding, v.4, p.399-417, 1940.

Hsu, S. M.; Ni, Chuen-Fa; Hung, Pi-Fang. Assessment of three infiltration formulas based on model fitting on Richards equations. Journal of Hydrologic Engineering, v.7, n.5, p.373-379, 2002.

Islam, N.; Wallender, W. W.; Mitchell, J. P.; Wicks, S.; Howitt, R. E. Performance evaluation of methods for the estimation of soil hydraulic parameters and their suitability in a hydrologic model, Geoderma, v.134, n.1-2, p.135-151, 2006.

Mls, J. Effective rainfall estimation. Journal of Hydrology, v.45, n.3, p.305-311, 1980.
Mollerup, M.; Hansen, S. Power series solution for falling head ponded infiltration with evaporation. Water Resources Research, v.43, p.W03425, 2007.

Mollerup, M.; Hansen, S.; Peterson, C.; Kjaersgaard, J. H. A MATLAB program for estimation of unsaturated hydraulic soil parameters using an infiltrometer technique. Computers \& Geosciences, v.34, n.8, p.861-875, 2008.

Morel-Seytoux, H. T. Derivation of equations of variable rain infiltration. Water Resources Research, v.14, n.4, p.561-568, 1978.

Omuto, C. T.; Gumbe, L. O. Estimating water infiltration and retention characteristics using a computer program in R. Computers \& Geosciences, v.35, n.3, p.579-585, 2009.

Shi, Zhi-Hua; Chen, Li-Ding; Fang, Nu-Fang; Qin, De-Fu; Cai, Chong-Fa. Research on the SCS-CN initial abstraction ratio using rainfall-runoff event analysis in the Three Gorges Area, China. Catena, v.77, n.1, p.1-7, 2009.

Smith, R. E.; Parlange, J. Y. A parameter efficient hydrologic infiltration model. Water Resources Research, v.14, n.3, p.533-538, 1978.

Tan, B. Q; O’Connor, K. M. Application of an empirical infiltration equation in the SMAR conceptual model. Journal of Hydrology, v.185, n.4, p.275-295, 1996.

Wang, Quan-Jiu; Horton, R.; Fan, Jun. An Analytical Solution for One-Dimensional Water Infiltration and Redistribution in Unsaturated Soil. Pedosphere, v.19, n.1, p.104-110, 2009.

Weill, S.; Mouche, E.; Patin, J. A generalized Richards equation for surface/subsurface flow modeling. Journal of Hydrology, v.366, n.1, p.9-20, 2009. 